\title{
Optical Signal Processing with Electrooptic Modulators and Dispersion
}

\author{
S. Tainta ${ }^{* 1}$, M.J. Erro ${ }^{1}$, M.J. Garde ${ }^{1}$, M.A. Muriel $^{2}$, S. Sales ${ }^{3}$ \\ *1 Dept. of Electric and Electronic Engineering, Universidad Publica de Navarra, 31006 Pamplona, Spain \\ ${ }^{2}$ Dept. of Photonic Technology, Polytechnic University of Madrid, Madrid, Spain \\ ${ }^{3}$ ITEAM Research Institute, Polytechnic University of Valencia, Valencia, Spain \\ Tel: (34) 948169328, Fax: (34) 948169720, e-mail: santiago.tainta@unavarra.es
}

\begin{abstract}
A review of the main techniques that have been proposed for temporal processing of optical pulses that are the counterpart of the well-known spatial arrangements will be presented. They are translated to the temporal domain via the space-time duality and implemented with electrooptical phase and amplitude modulators and dispersive devices. We will introduce new variations of the conventional approaches and we will focus on their application to optical communications systems.
\end{abstract}

Keywords: optical signal processing, second order dispersion, electrooptic modulation, linearly chirped fibre Bragg gratings.

\section{INTRODUCTION}

Optical signal processing finds several important applications, such as optical communications, nonlinear optics, coherent quantum control or femtosecond microscopy and spectroscopy, which explains the interest and development of this area in recent years.

One of the first and more popular setups for the shaping of optical signals is based on volume optics [1]. A bulk diffraction grating performs a spatial Fourier transform of the signal to be processed, so that each of its frequency components is found in a different point of the plane where a spatial light modulator is placed. This modulator conveniently modifies the phase and intensity of the frequency components of the signal, that are then recombined in another grating that performs an inverse Fourier transform to give the output signal with the desired temporal shape.

To avoid the drawbacks associated to spatial arrangements, a dual configuration in the temporal domain has also been proposed [2]. The fundamental of this alternative is the well-known analogy between the equations that describe the paraxial diffraction of beams in space and the dispersion of narrow-band pulses in dielectrics [3]. Based on this duality it was shown that a dispersive device, usually a Linearly Chirped-Fiber Bragg Grating (LCFBG), can be used to obtain the Fourier transform of a signal (in modulus only) [4] in the time domain. Electrooptic modulators are then used as the counterpart of the spatial light modulators to act as phase or amplitude filters on the Fourier domain. In this setup, the target is the shape of each individual optical pulse. And the limit is the repetition rate of the pulse sequence, since the temporal Fourier transform implies dispersion and, hence, pulse broadening, while simultaneously requiring that dispersed pulses do not overlap with neighbouring pulses.

Another proposal based on the space-time duality was the temporal self imaging effect [5], which is equivalent to the spatial Talbot effect, achieved by propagation of a periodic train of pulses through a single dispersive device (an optical fiber or a LC-FBG operated in reflection). This technique permits the multiplication of the repetition rate of the pulse train by an integer factor that is fixed by the value of the applied dispersion. The main limitation of this approach is the lack of programmability, since the dispersive device is designed to work only for a specific input-output pulse sequence pair.

We have recently proposed a new method that can be considered as a hybrid of the two mentioned temporal techniques [6]. It combines an electrooptic modulator with a single dispersive device and permits the shaping of a train of optical pulses, allowing the electrically tunable repetition rate multiplication, the control of the train envelope shape or a tunable delay of the optical pulse train, as will be explained in the next sections.

Finally, a new technique to obtain the spectral Talbot effect, the counterpart of the temporal Talbot effect in the frequency domain, by applying a multilevel time phase modulation is presented [7]. This allows the shaping of the optical comb at the output of the system, permitting the tunable control of the spacing between the different spectral lines.

\section{PROPOSED TECHNIQUE}

A general scheme of the proposed technique is shown in Fig. 1b. The signal to be processed by the system is a periodic train of short optical pulses with a repetition rate $f_{0}=1 / T_{0}$, where $T_{0}$ is the delay between two consecutive pulses, as in the conventional pulse train shaping technique represented in Fig. 1a. 
In the conventional approaches (Fig. 1a) the spectrum of the input signal is periodically filtered. The filtering can be applied in amplitude, in phase or in both depending on the processing desired. As an example, multiplication of the repetition rate by an integer factor $N$ can be obtained both by letting pass to the output only one of every $N$ spectrum lines when using amplitude filters or, if the filtering is in phase, by applying the phase shifts associated to each spectral component determined by the temporal fractional Talbot effect [5]. However, to process the individual spectral lines, high resolution optical filters are necessary, limiting thus these techniques by the available filtering resolution.

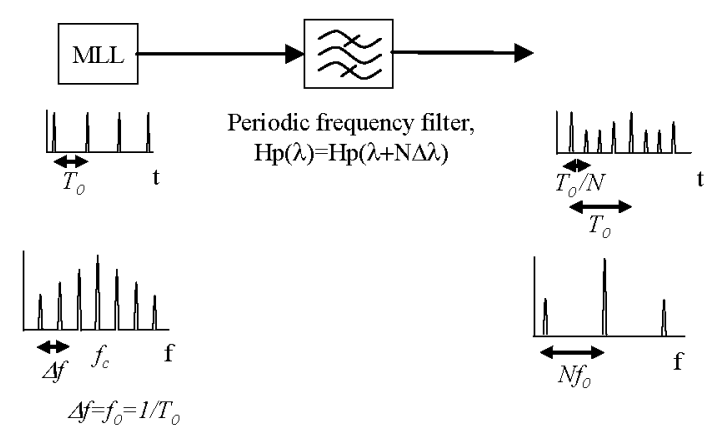

(a)

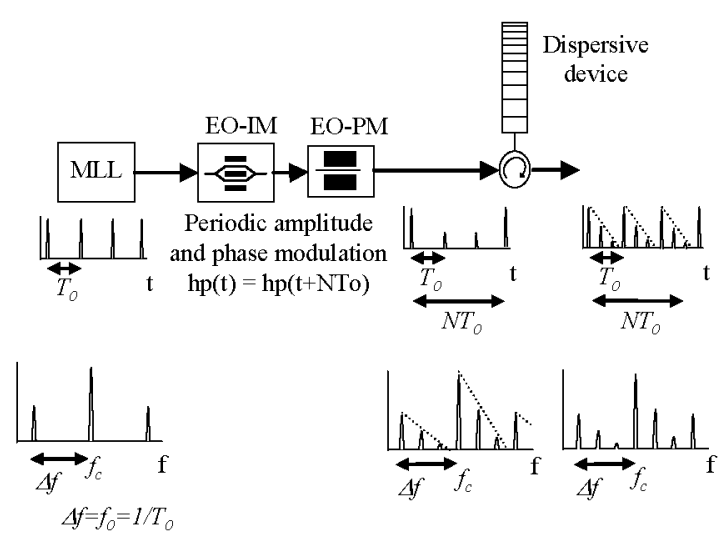

(b)

Figure 1: (a) Proposed technique with shaping in the time domain followed by a dispersive device. (b) Conventional technique with shaping in the frequency domain. MLL: Mode Locked Laser, EO-IM: Electrooptic Intensity Modulator, EO-PM: Electrooptic Phase Modulator.

The proposed technique is based on the manipulation of a periodic train of pulses on the time domain and a controlled interference between them caused by a dispersive device. As shown in Fig. 1b, the input train of pulses is processed in phase, amplitude or both by using electrooptical modulation in the time domain. The modulator signal $m(t)$ is chosen to be periodic with period $N T_{0}$ and as the dual of the periodic filter of Fig. 1a, imposing a periodic modulation. Also, as the pulse width of the pulses can be considered much faster than the time variations of the modulating signal, the modulation applied can be assumed to be constant during the whole duration of the individual pulses. Therefore, the modulated signal can be expressed as:

$$
x(t)=\sum_{l=0}^{N-1} m\left(l T_{0}\right) \sum_{k=-\infty}^{\infty} a\left(t-l T_{0}-k N T_{0}\right) .
$$

In (1) it can be observed that the time-modulated pulse sequence is the superposition of $N$ trains of pulses, where each of them has a period of $N T_{0}$ and is multiplied by a complex coefficient $m_{l}=m\left(l T_{0}\right)$. Also, an additional time-shift of $T_{0}$ appears between the consecutive trains. As demonstrated in [5], a temporal fractional Talbot effect with $s=1, m=N^{2}$ occurs when a train of pulses of period $N T_{0}$ passes through a dispersive device with dispersion given by:

$$
\ddot{\Phi}=\left.\frac{s\left(N T_{0}\right)^{2}}{m 2 \pi}\right|_{s=1, m=N^{2}}=\frac{T_{0}^{2}}{2 \pi} .
$$

That is, when the modulated sequence is dispersed, each of the $N$ sub-trains of pulses results in another pulse train with a period of $N T_{0} / N^{2}=T_{0} / N$ that superposes coherently with the rest of incident trains. As a result, the average optical power of the signal at the output of the system is

$$
P(t) \propto \sum_{k^{\prime}=-\infty}^{\infty}\left|n_{k^{\prime}}\right|^{2}\left|a\left(t-k^{\prime} \frac{T_{0}}{N}-\frac{T_{0}}{2}\right)\right|^{2},
$$

which corresponds to a train of pulses with a repetition factor $T_{0} / N$ as expected, where the amplitude of the pulses is determined by a set of coefficients that depend on the modulating signal. Also, an additional time shift of $T_{0} / 2 N$ appears, depending on $N$ being even or odd. The relation between the modulation coefficients $m_{l}$ and the output envelope coefficients is given by:

$$
n_{k^{\prime}}=\left\{\begin{array}{cc}
\frac{1}{N^{2}} \sum_{l=0}^{N-1} m_{l} \sum_{q=0}^{N^{2}-1} \exp \left(j \frac{\pi}{N^{2}}\left\{q^{2}+2\left(k^{\prime}-l N\right) q\right\}\right) & \text { N even } \\
\frac{1}{N^{2}} \sum_{l=0}^{N-1} m_{l} \sum_{q=0}^{N^{2}-1} \exp \left(j \frac{\pi}{N^{2}}\left\{q^{2}+2\left(k^{\prime}-l N+1\right) q\right\}\right) & \mathrm{N} \text { odd }
\end{array} .\right.
$$




\section{APPLICATIONS}

\subsection{Shaping of optical trains of pulses}

The most straightforward application of the technique is the simultaneous control of the amplitude envelope and the repetition rate of an optical train of pulses [6]. By controlling the periodicity of the modulated signal, $N T_{0}$, different repetition rates can be obtained at the output of the system. Additionally, the individual amplitude of each one of the pulses, $\left|n_{k^{\prime}}\right|$, can be controlled through the modulating signal coefficients $m_{l}$. These coefficients can be calculated by using an optimization algorithm to minimize:

$$
F\left(m_{0}, \ldots, m_{N-1}\right)=\sum_{k^{\prime}=0}^{N-1}|| n_{k^{\prime}}\left|-y_{k^{\prime}}\right|,
$$

where $y_{k^{\prime}}$ are the desired amplitudes for the different pulses in one period of the output signal. If an arbitrary envelope of the output train of pulses is to be obtained, both amplitude and phase manipulation of the input pulses are required. However, phase-only shaping is preferred in some cases such as RRM because of its greater energetic efficiency, requiring also the use of only one electro-optic phase modulator to perform the shaping. Also, the generation of a multilevel modulating signal requires the use of a RF arbitrary waveform generator. Therefore, for simplicity only the case of bilevel phase modulation will be considered. Figure 2 shows simulation results for the train of pulses at the outputs for different repetition ratios and different envelopes when an input train of pulses with a repetition rate of $10 \mathrm{GHz}$ is used.

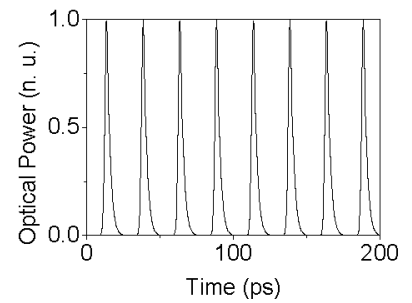

(a)

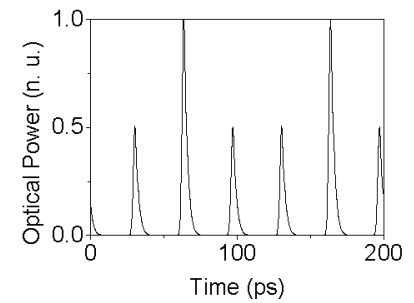

(b)

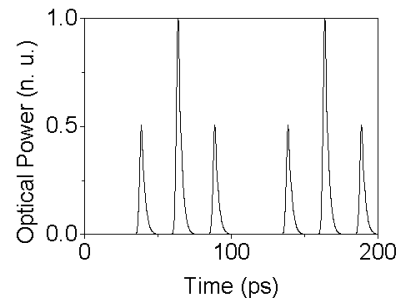

(c)

Figure 2. Trains of pulses at the output of the system for (a) $N=4$ and uniform envelope

(b) $N=4$ and a triangular envelope and (c) $N=5$ and a triangular envelope.

\subsection{Optical delay line}

A different application of the proposed technique is the realization of an optical delay line. When a linear phase is applied before the dispersive device, it can be demonstrated that the output train of pulses suffers a delay proportional to the slope of the applied signal. In order to introduce this modulation, an electrooptic phase modulator can be used, applying to its input an electrical linear signal. However, it is not possible to generate a linear electric signal, except in a finite time window. To overcome this limitation, the proposed system uses a ramp signal with a peak to peak voltage equal to $2 V_{\pi}$, where $V_{\pi}$ is the half-wave voltage of the phase modulator. Due to the periodicity of the phase modulation, this signal is equivalent to a linear phase modulation, but it also limits the possible periodicity of the signal to multiples of the input repetition rate $\left(T_{1}=k T_{0}\right)$. Under these assumptions, the delay introduced by the system is:

$$
\Delta t=\frac{T_{0}}{2}\left(1 \pm \frac{2}{k}\right)
$$

where the sign of the delay introduced depends on the slope of the ramp signal used. Simulation results are shown in Fig. 3 for different frequencies of the ramp signal. As it can be observed, although the achievable delays are limited by the possible values of $k$, by combining the positive and negative slope and depending on the frequency of the ramp signal applied, a wide tunability range can be achieved over the whole period range.

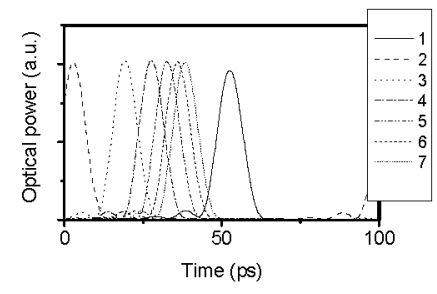

(a)

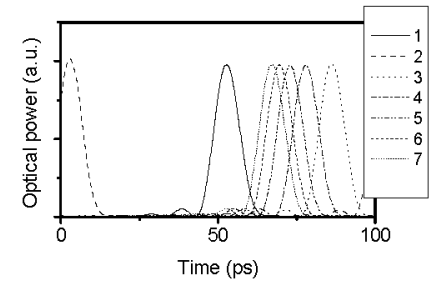

(b)

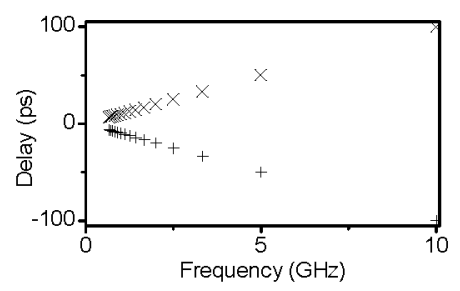

(c)

Figure 3. Delayed pulses at the output of the system for different values of $k$ when negative slope (a) and positive slope (b) are used. (c) Delay introduced for different frequencies of the ramp signal applied. 


\subsection{Spectral Talbot effect}

As it has been shown in the previous sections, the temporal Talbot effect can be used in combination with electro-optic modulation for the shaping of optical trains of pulses. However, the spectrum of the signal is not controllable by using these methods. Recently, an equivalent version to the temporal Talbot effect but that works in the spectral domain has been proposed [7]. The technique is based on the application of a multi-level phase modulation in the time domain to an input train of pulses, applying to each input pulse a phase shift whose value is determined by:

$$
\varphi_{n}= \pm \frac{s}{r} \pi n^{2}
$$

Simulation results for the proposed technique are shown in Fig. 4 when only bilevel phase modulation is used. Depending on the election of the parameters $s$ and $r$, different combs can be obtained at the output of the system where the spacing between the different frequency lines can be selected. As an example, Fig. $4 \mathrm{c}$ corresponds to an inverted integer spectral Talbot effect, producing a frequency shift of $f_{0} / 2$ of the spectral lines. Also, in Fig. $4 \mathrm{~d}$ the parameters selected were $s=1$ and $r=2$, resulting in a frequency comb where the spacing between the spectral lines is reduced by a factor of $r$.

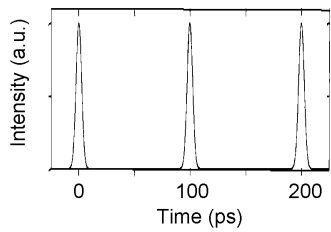

(a)

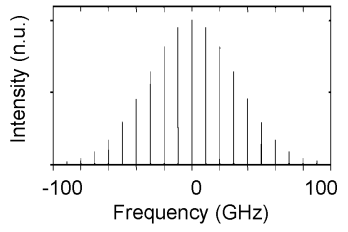

(b)

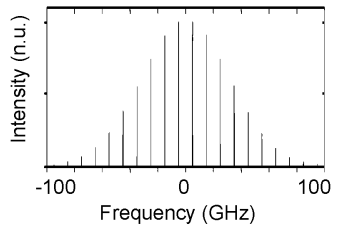

(c)

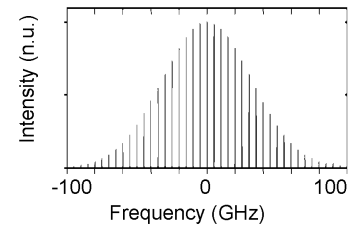

(d)

Figure 4. Simulation results of the spectral Talbot effect: (a) Input train of pulses and (b) spectrum of the input train of pulses. Output frequency comb after time modulation with (c) $s=1, r=1$ and (d) $s=1, r=2$.

\section{CONCLUSIONS}

We have reviewed the fundamentals and applications of a new technique that combines electrooptic modulation and a dispersive device to manipulate both the repetition rate and the repetition rate of a train of periodic optical pulses. It has been shown that, by properly choosing the electrical signal that is applied to the modulator, different effects can be obtained, such as the shaping of the train of pulses or the realization of an electrically tunable optical delay line. Also, a new method to perform the spectral Talbot effect has been presented that can potentially allow the use of the proposed technique also to shape the spectrum of optical trains of pulses.

\section{ACKNOWLEDGEMENTS}

The authors wish to acknowledge the financial support from the Ministerio de Ciencia e Innovacion of Spain under Projects TEC2007-68065-C03-01-02-03 and TEC2010-21305-C04-01 and -02.

\section{REFERENCES}

[1] A. M. Weiner, et al.: Shaping of femtosecond pulses using phase-only filters designed by simulated annealing, J. Opt. Soc. Am. A, vol. 10, pp. 1112-1120, May 1993.

[2] S. Thomas, et al.: Fiber-based programmable picosecond optical pulse shaper, J. Lightwave Technol., vol. 28, pp. 1832-1843, 15 Jun. 2010.

[3] B. H. Kolner: Space-time duality and the theory of temporal imaging, IEEE J. Quantum. Electron., vol. 30, pp. 1951-1963, Aug. 1994

[4] M. A. Muriel, J. Azaña, and A. Carballar: Real-time Fourier transformer based on fiber gratings, Opt. Lett., vol. 24, pp. 1-3, Jan. 1999.

[5] J. Azaña and M. A. Muriel: Temporal self-imaging effects: theory and application for multiplying pulse repetition rates, IEEE J. Sel. Topics Quantum Electron., vol. 7, pp. 728-744, Jul. 2001.

[6] S. Tainta, et al.: Periodic time-domain modulation for the electrically tunable control of optical pulse train envelope and repetition rate multiplication, to be published on IEEE J. Sel. Topics Quantum Electron., Sep. 2011

[7] J. Caraquitena, et al.: Spectral self-imaging effect by time-domain multilevel phase modulation of a periodic pulse train, Opt. Let., vol. 36, pp. 858-860, 15 Mar. 2011. 